\title{
Uniqueness of Bounded Variation Solutions for Measure Functional Differential Equations with Infinite Delay
}

\author{
Baolin Li, Yinxing Yang
}

Abstract-By using Henstock-Kurzweil integral, the uniqueness theorems of bounded variation solutions for measure functional differential equations with infinite delay are established. This result generalizes theorem concerning uniqueness in Lebesgue integral setting to a Henstock -Kurzweil integral setting.

Index Terms - measure functional differential equations with infinite delay; Henstock-Kurzweil integral; bounded variation solution; uniqueness.

\section{INTRODUCTION}

The Henstock-Kurzweil integral encompasses the Riemann and Lebesgue integrals [1]. the integral was introduced by Henstock and Kurzweil independently in 1957-1958 and was proved useful in the study of ordinary differential equations (see [2]).

Measure functional differential equations with infinite delay have the form

$$
\left\{\begin{array}{l}
x(t)=x\left(t_{0}\right)+\int_{t_{0}}^{t} f\left(x_{s}, s\right) d g(s), \\
x\left(t_{0}\right)=\phi
\end{array}\right.
$$

which have been introduced in the paper [3] by slavík, where $x$ is an unknown function with values in $R^{n}$ and the symbol $x_{s}$ denotes the function $x_{s}(\tau)=x(s+\tau)$ defined on $[-r, 0], r \geq 0$ being a fixed number corresponding to the length of the delay. The integral on the right-hand side of (1) is the Kurzweil-Stieltjes integral with respect to a nondecreasing function $g$. we consider that the integrands $f$ is Henstock-Kurzweil integrable and $\phi$ is a regulated function.

Let $G\left([a, b], R^{n}\right)$ be the space of regulated functions $x:[a, b] \rightarrow R^{n}$, that is, the lateral limits

$$
x(t+)=\lim _{\rho \rightarrow 0_{+}} x(t+\rho), t \in[a, b)
$$

and

$$
x(t-)=\lim _{\rho \rightarrow 0_{-}} x(t+\rho), t \in(a, b],
$$

exist and are finite. $G\left([a, b], R^{n}\right)$ which is a Banach

Baolin Li, College of Mathematics and Statistics, Northwest Normal University,LanzhouGansu730070,China.

Yinxing Yang, College of Mathematics and Statistics, Northwest Normal University, Lanzhou Gansu 730070,China. space when endowed with the norm $\|\phi\|=\sup _{a \leq t \leq b}\|\phi(t)\|$ for all $\|\phi\| \in G\left([a, b], R^{n}\right)$. Also, any function in $G\left([a, b], R^{n}\right)$ is the uniform limit of step functions. Define

$$
\begin{gathered}
G^{-}\left([a, b], R^{n}\right)=\left\{u \in G\left([a, b], R^{n}\right): u\right. \text { is left } \\
\text { continuous at every } t \in(a, b]\} .
\end{gathered}
$$

In $G^{-}\left([a, b], R^{n}\right)$, we consider the norm induced by $G^{-}\left([a, b], R^{n}\right)$. We denote by $B V\left([a, b], R^{n}\right)$ the space of functions $x:[a, b] \rightarrow R^{n}$ which are of bounded variation. In $B V\left([a, b], R^{n}\right)$, we consider the variation norm given by $\|x\|_{B V}=\|x(a)\|+\operatorname{Var}_{a}^{b} x$, where $\operatorname{Var}_{a}^{b} x$ Stands for the variation of $x$ in the interval $[a, b]$. Then $\left(B V\left([a, b], R^{n}\right),\|\cdot\|_{B V}\right)$ is a Banach space and $B V([a$ $\left.b], R^{n}\right) \subset G\left([a, b], R^{n}\right)$. When $x \in B V\left([a, b], R^{n}\right)$ is also left continuous, we write $x \in B V^{-}\left([a, b], R^{n}\right)$.

It is clear that for a function $x \in G^{-}\left(\left[-\infty, t_{0}+\sigma\right]\right.$, $\left.R^{n}\right)$, we have $x_{t} \in G^{-}\left([-r, 0], R^{n}\right)$ for all $t \in\left[t_{0}, t_{0}\right.$ $+\sigma]$.

Let $x \in G^{-}\left(\left[-\infty, t_{0}+\sigma\right], R^{n}\right)$ with the following property: if $x=x(t), t \in\left(-\infty, t_{0}+\sigma\right]$, is an element of $G_{1}$ and $\bar{t} \in\left(-\infty, t_{0}+\sigma\right]$, then $\bar{x}$ given by

$$
\bar{x}(t)= \begin{cases}x(t), & -\infty \leq t \leq \bar{t} \\ x(\bar{t}+), & \bar{t}<t \leq t_{0}+\sigma\end{cases}
$$

Also belongs to $G_{1}$.

Let $H_{1} \in G^{-}\left([-r, 0], R^{n}\right)$ be such that

$$
\left\{x_{t} \mid t \in\left[t_{0}, t_{0}+\sigma\right], x \in G_{1}\right\} \subset H_{1} .
$$

The existence of bounded variation solutions for measure functional differential equation with infinite delay were obtained in [4]. On this basis, the uniqueness of bounded variation solutions for measure functional differential equation 
with infinite delay is discussed in this paper.

\section{PRELIMINARIES}

Let $[a, b]$ be a compact interval in $\mathrm{R}$ and $\|\cdot\|$ be a norm in $R^{n}$.

Let $\delta(\xi)$ be a positive function on $[a, b]$, i.e.

$\delta(\xi):[a, b] \rightarrow R^{+}$. We say $D=\left\{\left(\left[t_{i-1}, t_{i}\right], \xi_{i}\right)\right\}_{i=1}^{m}$ is $\delta$-fine of $[a, b]$ if $\xi_{i} \in\left[t_{i-1}, t_{i}\right] \subset\left(\xi_{i}-\delta\left(\xi_{i}\right), \xi_{i}+\right.$ $\left.\delta\left(\xi_{i}\right)\right)$ for all $i=1,2, \cdots, n$.

Definition. ${ }^{[1]}$

A function $u:[a, b] \rightarrow R^{n}$ is said to be

Henstock-Kurzweil integrable on $[a, b]$ if there exists an $I \in R^{n}$ such that for every $\varepsilon>0$, there exists $\delta(\xi):[a, b] \rightarrow R^{+}$such that for every $\delta$-fine partition $D=\left\{\left(\left[t_{i-1}, t_{i}\right], \xi_{i}\right)\right\}_{i=1}^{m}$, we have

$$
\left\|\sum_{i=1}^{m} u\left(\xi_{i}\right)\left(t_{i}-t_{i-1}\right)-I\right\|<\varepsilon .
$$

We denote the Henstock-Kurzweil integral (also write as $\mathrm{H}-$ $\mathrm{K}$ integral) $I$ by $(\mathrm{H}-\mathrm{K}) \int_{a}^{b} u(s) d s$.

Definition. $2^{[4]}$

Assume $f\left(x_{t}, t\right): H_{1} \times\left[t_{0}, t_{0}+\sigma\right] \rightarrow R^{n}$ satisfy the following conditions:

(A) Then exists a positive function $\delta(\tau):\left[t_{0}, t_{0}+\sigma\right] \rightarrow$ $R^{+}$such that for every $[u, v]$ satisfy $\tau \in[u, v] \subset(\tau-$ $\delta(\tau), \tau+\delta(\tau)) \subset\left[t_{0}, t_{0}+\sigma\right]$ and $x \in G_{1}$, we have

$$
\left\|f\left(x_{\tau}, \tau\right)(g(v)-g(u))\right\|<|h(v)-h(u)| .
$$

(B) For every $[u, v]$ satisfy $\tau \in[u, v] \subset(\tau-\delta(\tau), \tau+$ $\delta(\tau)) \subset\left[t_{0}, t_{0}+\sigma\right]$ and $x, y \in G_{1}$, we have

$$
\begin{gathered}
\left\|f\left(x_{\tau}, \tau\right)-f\left(y_{\tau}, \tau\right)\right\|(g(v)-g(u))< \\
\omega\left(\left\|x_{\tau}-y_{\tau}\right\|\right)|h(v)-h(u)|,
\end{gathered}
$$

where $h:\left[t_{0}, t_{0}+\sigma\right] \rightarrow R$ is a nondecreasing function and continuous the left. $\omega:[0, \infty] \rightarrow R$ is a continuous and increasing function with $\omega(0)=0, \omega(r)>0$ for $r>0$.

\section{Definition. $3^{[4]}$}

Let $\Omega \subset H_{1} \times\left[t_{0}, t_{0}+\sigma\right]$ be open. Assume that function $f: \Omega \rightarrow R^{n}$ is a Caratheodory function and belongs to the class $W(\Omega, h, \omega)$ if $f$ satisfys the condition (A), (B).

\section{Lemma.1 $^{[5]}$}

Assume that $\psi:[a, b] \rightarrow[0,+\infty)$ is a bounded function on $[a, b], h:[a, b] \rightarrow[0,+\infty)$ is a nondecreasing function and continuous from the left on the interval $[a, b]$, $\omega:[0,+\infty] \rightarrow R$ is a continuous and increasing function with $\omega(0)=0, \omega(r)>0$ for $r>0$. Assume

$$
F(u)=\int_{u_{0}}^{u} \frac{1}{\omega(r)} d r,
$$

for $u>0$, where $u_{0}>0 . F:[0, \infty] \rightarrow R$ is a increasing function, $F\left(u_{0}\right)=0$ and

$$
\lim _{u \rightarrow 0_{+}} F(u)=\alpha \geq-\infty, \quad \lim _{u \rightarrow+\infty} F(u)=\beta \leq+\infty .
$$

Assume the inequality

$$
\psi(\xi) \leq k+\int_{a}^{\xi} \omega(\psi(t)) d h(t)
$$

holds for $\xi \in[a, b]$, where $k$ is a constant and $k>0$.

If $F(k)+h(b)-h(a)<\beta$, then the inequality

$$
\psi(\xi) \leq F^{-1}[F(k)+h(\xi)-h(a)]
$$

holds for $\xi \in[a, b]$, where $F^{-1}:[\alpha, \beta] \rightarrow R$ is the inverse function to $F$ form (2).

\section{PRIME RESULT}

\section{Definition.1}

A bounded variation solution $x:\left[-\infty, t_{0}+\eta\right] \rightarrow R^{n}$ of (1) is said to be locally unique for increasing values of $t$ if for any solution $y:\left[-\infty, t_{0}+\alpha\right] \rightarrow R^{n}, \alpha>0$ of (1) with $y_{t_{0}}=x_{t_{0}}=\phi$ there exists $\eta_{1}>0$ such that $x(t)=y(t)$ for $t \in\left[-\infty, t_{0}+\eta\right] \cap\left[-\infty, t_{0}+\alpha\right] \cap\left[-\infty, t_{0}+\eta_{1}\right]$.

\section{Theorem.1}

Assume that $f \in W(\Omega, h, \omega)$, where $h$ is a function and continuous from the left, $\omega:[0,+\infty) \rightarrow R$ is a continuous and increasing function with $\omega(0)=0$, $\omega(r)>0$ for $r>0$, and for $u>0$, we have

$$
\lim _{v \rightarrow 0+} \int_{v}^{u} \frac{1}{\omega(r)} d r=+\infty .
$$

Then every bounded variation solution $x=x(t)$ with $x_{t_{0}}=\phi$ of (1) is locally unique for increasing values of $t$, $\left(t_{0}, \phi\right) \in \Omega$.

Proof Assume that $x, y:\left[-\infty, t_{0}+\eta\right] \rightarrow R^{n}$ are two bounded variation solution of (1) with $x_{t_{0}}=y_{t_{0}}=\phi$, where $\eta>0$. Then 


$$
\begin{aligned}
\|x(t)-y(t)\| & =\left\|\int_{t_{0}}^{t}\left[f\left(x_{s}, s\right)-f\left(y_{s}, s\right)\right] d g(s)\right\|, \\
t_{0} & \leq t \leq t_{0}+\eta
\end{aligned}
$$

For arbitrary $\varepsilon>0$ there exists a positive function $\delta(\tau):\left[t_{0}, t\right] \rightarrow R^{+}$, such that for any $\delta(\tau)$-fine partition $D=\left\{\left(\left[t_{i-1}, t_{i}\right], \tau_{i}\right)\right\}_{i=1}^{m}$. By Definition 1 and condition (B), we have

$$
\begin{gathered}
\left\|\int_{t_{0}}^{t}\left[f\left(x_{s}, s\right)-f\left(y_{s}, s\right)\right] d g(s)\right\| \leq \\
\| \int_{t_{0}}^{t}\left[f\left(x_{s}, s\right)-f\left(y_{s}, s\right)\right] d g(s)- \\
\sum_{i=1}^{m}\left[f\left(x_{\tau_{i}}, \tau_{i}\right)-f\left(y_{\tau_{i}}, \tau_{i}\right)\right]\left(g\left(t_{i}\right)-g\left(t_{i-1}\right)\right) \|+ \\
\left\|\sum_{i=1}^{m}\left[f\left(x_{\tau_{i}}, \tau_{i}\right)-f\left(y_{\tau_{i}}, \tau_{i}\right)\right]\left(g\left(t_{i}\right)-g\left(t_{i-1}\right)\right)\right\|< \\
\frac{\varepsilon}{2}+\sum_{i=1}^{m} \omega\left(\left\|x_{\tau_{i}}-y_{\tau_{i}}\right\|\right)\left(h\left(t_{i}\right)-h\left(t_{i-1}\right)\right) .
\end{gathered}
$$

Owing to

$$
\begin{gathered}
\sum_{i=1}^{m} \omega\left(\left\|x_{\tau_{i}}-y_{\tau_{i}}\right\|\right)\left(h\left(t_{i}\right)-h\left(t_{i-1}\right)\right) \leq \\
\| \sum_{i=1}^{m} \omega\left(\left\|x_{\tau_{i}}-y_{\tau_{i}}\right\|\right)\left(h\left(t_{i}\right)-h\left(t_{i-1}\right)\right)- \\
\int_{t_{0}}^{t} \omega\left(\left\|x_{s}-y_{s}\right\|\right) d h(s) \|+ \\
\int_{t_{0}}^{t} \omega\left(\left\|x_{s}-y_{s}\right\|\right) d h(s)< \\
\frac{\varepsilon}{2}+\int_{t_{0}}^{t} \omega\left(\left\|x_{s}-y_{s}\right\|\right) d h(s) .
\end{gathered}
$$

By (5)-(7), we get

$$
\begin{aligned}
\|x(t)-y(t)\|= & \left\|\int_{t_{0}}^{t}\left[f\left(x_{s}, s\right)-f\left(y_{s}, s\right)\right] d g(s)\right\| \\
& <\varepsilon+\int_{t_{0}}^{t} \omega\left(\left\|x_{s}-y_{s}\right\|\right) d h(s) \\
& <\int_{t_{0}}^{t} \omega\left(\left\|x_{s}-y_{s}\right\|\right) d h(s) \\
& =\int_{t_{0}}^{t_{0}+\delta} \omega\left(\left\|x_{s}-y_{s}\right\|\right) d h(s)+ \\
& \int_{t_{0}+\delta}^{t} \omega\left(\left\|x_{s}-y_{s}\right\|\right) d h(s),
\end{aligned}
$$

Where $0<\delta<t-t_{0}$.

According to [5], we obtain

$$
\begin{gathered}
\int_{t_{0}+\delta}^{t} \omega\left(\left\|x_{s}-y_{s}\right\|\right) d h(s)= \\
\omega\left(\left\|x_{t_{0}}-y_{t_{0}}\right\|\right)\left[h\left(t_{0}+\right)-h\left(t_{0}\right)\right]+
\end{gathered}
$$

$$
\lim _{t_{1} \rightarrow t_{0}^{+}} \int_{t_{1}}^{t_{0}+\delta} \omega\left(\left\|x_{s}-y_{s}\right\|\right) d h(s) \leq
$$$$
\sup _{s \in\left(t_{0}, t_{0}+\delta\right]} \omega\left(\left\|x_{s}-y_{s}\right\|\right)\left(h\left(t_{0}+\delta\right)-h\left(t_{0}\right)\right)=A(\delta) \text {, }
$$

becasure of $h\left(t_{0}+\right)$ exists, then $\lim _{\delta \rightarrow 0^{+}} A(\delta)=0$. Thus

$$
\|x(t)-y(t)\| \leq A(\delta)+\int_{t_{0}+\delta}^{t} \omega\left(\left\|x_{s}-y_{s}\right\|\right) d h(s),
$$

where $t \in\left[t_{0}+\delta, t_{0}+\eta\right]$.

For given $u_{0}>0$, set $F(u)=\int_{u_{0}}^{u} \frac{1}{\omega(r)} d r$. Then the imequality

$$
\|x(t)-y(t)\| \leq F^{-1}\left(F A(\delta)+h(t)-h\left(t_{0}+\delta\right)\right)
$$

holds, where

$$
\begin{gathered}
t \in\left[t_{0}+\delta, t_{0}+\eta\right], F(A(\delta))+h(t)-h\left(t_{0}+\delta\right)<\beta, \\
\beta=\lim _{u \rightarrow+\infty} F(u) \leq+\infty .
\end{gathered}
$$

Obviously, we have

$$
\begin{gathered}
F(A(\delta))+h\left(t_{0}+\eta\right)-h\left(t_{0}+\delta\right) \leq \\
F(A(\delta))+h\left(t_{0}+\eta\right)-h\left(t_{0}+\right),
\end{gathered}
$$

and for

$$
\lim _{\delta \rightarrow 0^{+}} A(\delta)=0, \quad \lim _{u \rightarrow 0^{+}} F(u)=-\infty .
$$

Hence

$$
\lim _{\delta \rightarrow 0^{+}} F(A(\delta))+h\left(t_{0}+\eta\right)-h\left(t_{0}+\right)=-\infty .
$$

Therefore there exists $\delta_{0}>0$ such that for $\delta \in\left(0, \delta_{0}\right)$ the inequality $F(A(\delta))+h\left(t_{0}+\eta\right)-h\left(t_{0}+\right)<\beta$ holds, and we get

$$
\begin{aligned}
F\left(\left\|x_{t}-y_{t}\right\|\right)-A(\delta) & \leq h(t)-h\left(t_{0}+\delta\right) \\
& \leq h(t)-h\left(t_{0}+\right) .
\end{aligned}
$$

According the definition of function $F$, for $t \in\left[t_{0}+\delta, t_{0}+\eta\right]$ and $\delta \in\left(0, \delta_{0}\right)$,we have

$$
\int_{A(\delta)}^{|x(t)-y(t)| \mid} \frac{1}{\omega(r)} d r \leq h\left(t_{0}+\eta\right)-h\left(t_{0}+\right) .
$$

If $\left\|x\left(t^{*}\right)-y\left(t^{*}\right)\right\|=k>0$ for some $t^{*} \in\left(t_{0}, t_{0}+\eta\right]$, then for $\delta \in\left(0, \delta_{0}\right)$ such that $\delta<t^{*}-t_{0}$, we have

$$
\int_{A(\delta)}^{k} \frac{1}{\omega(r)} d r \leq h\left(t_{0}+\eta\right)-h\left(t_{0}+\right)<+\infty,
$$

thus

$$
\lim _{\delta \rightarrow 0^{+}} \int_{A(\delta)}^{k} \frac{1}{\omega(r)} d r \leq h\left(t_{0}+\eta\right)-h\left(t_{0}+\right)<\infty,
$$

this is in contradiction with the function $\omega$, so $\|x(t)-y(t)\|=0$ for all $t \in\left[t_{0}, t_{0}+\eta\right]$. Therefore, the theorem is proved.

Corollary.1 
If $f \in W(\Omega, h, \omega)$, where $\omega(r)=L r, r \geq 0, L \geq 0$, then the bounded variation solution of (1) which satisfies $\left(t_{0}, \phi\right) \in \Omega$ is locally unique for increasing values of $t$.

Proof For $u>0$, obviously

$$
\lim _{v \rightarrow 0^{+}} \int_{v}^{u} \frac{1}{\omega(r)} d r=\lim _{v \rightarrow 0^{+}} \frac{1}{L} \ln \frac{u}{v}=+\infty,
$$

the conditions of theorem 3.1 are satisfied, then the corollary holds.

\section{REFERENCES}

[1] P. Y. Lee. Lanzhou Lectures on Henstock integration [M]. Singapore: World Scientific, 1989.

[2] J. Kurzweil. Genenralized ordinary differential equations and continuous dependence on a parameter [J]. Czechoslovak Math J,1957,82(7):418-448.

[3] Slavík A . Measure functional differential equations with infinite delay [J]. Nonl Anal,2013,79(1):140-155 .

[4] B. L. Li, Z. Y. Xu. Bounded variation solutions for measure functional differential equations with infinite delay $[\mathrm{J}]$. Journal of Gansu Sciences, 2019,31(2):1-7

[5] J. Kurzweil. Generalized Ordinary Differential Equations [M].1992.

[6] Y. Hino, S. Murakami, T. Naito. Functional differential equations with infinite delay [M]. New York:Springer-Verlag,1991

[7] M Federson, J G Mesquita, A Slavík. Measure functional differential equations and functional dynamic equations on time scales [J]. J Diff Eqns, 2012,252(6):3816-3847

[8] A Slavík. Well-posedness results for abstract generalized differential equations and measure functional differential equations. Journal of Differential Equations,2015,259(2):666-707. 